\section{CMOS low dropout regulator with dynamic zero compensation}

\section{C.-L. Chen, W.-J. Huang and S.-I. Liu}

\begin{abstract}
The output capacitor and its equivalent series resistance (ESR) often limit the stability of a conventional low dropout regulator (LDR). A CMOS LDR with dynamic zero compensation is presented to tolerate the wide range of the output capacitor and the ESR. The stability constraints for the output capacitor with the ESR are derived. The measure LDR is stable for the output capacitor $2 \mathrm{nF}-47 \mathrm{uF}$ with ESR of $0.1-50 \Omega$. The maximum quiescent current of this LDR with a bandgap reference is $43.2 \mathrm{uA}$ and its maximum output current is $150 \mathrm{~mA}$ for the output voltage of $1.8 \mathrm{~V}$.
\end{abstract}

Introduction: Low dropout regulators (LDRs) [1-4] are widely used in power management integrated circuits and systems. A conventional LDR needs a large output capacitor to reduce output ripples. However, the output capacitor and its equivalent series resistance (ESR) often affect the stability of the LDR. In [4], it is indicated that the allowable range of ESR is limited, such as $0.2-9 \Omega$, for a typical LDR. To extend the allowable range of the output capacitor and its ESR, an LDR with dynamic zero compensation is presented.

Circuit description: The proposed LDR with dynamic zero compensation is shown in Fig. 1 (the bandgap voltage reference is not shown) It is composed of three gain stages, a current follower $[3,5,6]$ with a Miller compensation capacitor $C_{a}$, and a feedback resistor network. The dynamic zero compensation is realised by a PMOS transistor, $M_{z}$, and a capacitor, $C_{z}$. It introduces a dynamic low-frequency zero, which is dependent on the output current, $I_{\text {out }}$. As the output current is zero, both the power PMOS transistor, $M_{p}$, and $M_{z}$ are turned off, and this zero is not active. As the output current is increased, $M_{p}$ and $M_{z}$ are turned on. Then, the low-frequency zero is realised to enable the pole-zero elimination. It extends the useful range of the output capacitor and the ESR for the proposed LDR.

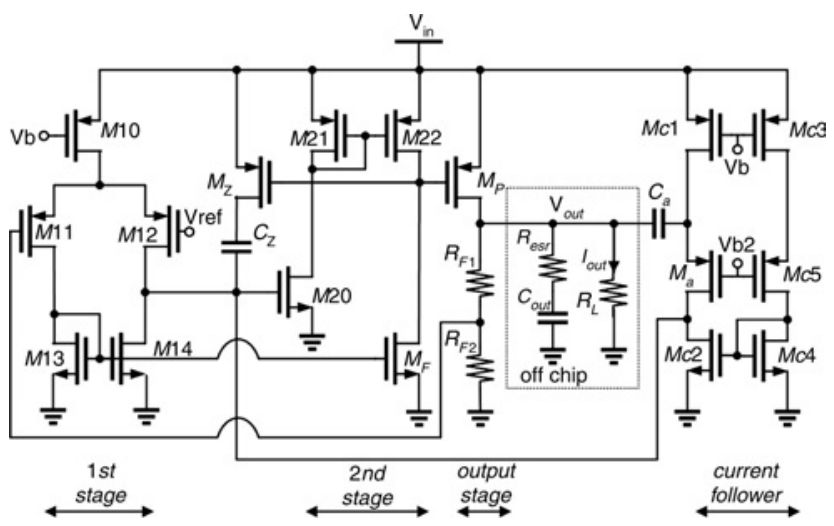

Fig. 1 Proposed LDR

To understand the design issues, the following analysis is derived. $g_{m 1}, g_{m 2}$ and $g_{m p}$, are the transconductances of the first, second and the output stage, respectively. $R_{1}, R_{2}$ and $R_{p}$ are output resistances of the first, second and the output stage, respectively. $\mathrm{C}_{1}$ and $\mathrm{C}_{2}$ are output parasitic capacitance of the first and second stage, respectively. $g_{m a}$ and $g_{m f}$ are the transconductances of the PMOS transistor, $M_{a}$, and the NMOS one, $M_{F}$, respectively. $R_{z}$ is the equivalent source-to-drain resistance of the transistor $M_{z} . C_{z}$ and $C_{a}$ are the compensation capacitors. $C_{o u t}$ is the output capacitor with the associated ESR, $R_{e s r}$. $R_{\text {out }}$ is the total equivalent output resistance, which is equal to $R_{L} / / R_{p}$ $/ /\left(R_{F 1}+R_{F 2}\right)$, where $R_{L}$ is the load resistor, and $R_{F 1}$ and $R_{F 2}$ are the feedback resistors.

Assume $C_{\text {out }} \gg\left(C_{z}, C_{a}, C_{2}\right) \gg C_{1}, g_{m p} \gg\left(g_{m 1}, g_{m 2}, g_{m a}, g_{m f}\right)$, and $\left(g_{m 1} R_{1}, g_{m 2} R_{2}, g_{m p} R_{\text {out }}\right) \gg 1$. Based on the above assumptions, the loop transfer function of the proposed LDR in the light load (i.e. $I_{\text {out }}$ is small) is given as

$$
\begin{aligned}
L_{l}(s) \simeq & \frac{-A_{d c}\left(1+s C_{\text {out }} R_{e s r}\right)\left(1+s\left(g_{m f} C_{1} / g_{m 1} g_{m 2}\right)\right)}{\left(1+\left(s / P_{1\lrcorner}\right)\right)\left(1+s\left(\left(C_{a} R_{1} g_{m 2} R_{2} g_{m p} R_{e s r}\right.\right.\right.} \\
& \left.\left.+C_{2} R_{2}+C_{1} R_{1}\right) /\left(C_{a} / C_{\text {out }}\right)\left(R_{1} g_{m 2} R_{2} g_{m p}+1\right)\right) \\
& \left.+s^{2}\left(\left(C_{1} R_{1} C_{2} R_{2}\right) /\left(C_{a} / C_{\text {out }}\right) R_{1} g_{m 2} R_{2} g_{m p}+1\right)\right)
\end{aligned}
$$

where $A_{d c}=\left(R_{F 2} /\left(R_{F 1}+R_{F 2}\right)\right) g_{m 1} R_{1} g_{m 2} R_{2} g_{m p} R_{\text {out }}$ is the DC loop gain and $p_{1-l}=1 /\left(C_{a} R_{1} g_{m 2} R_{2} g_{m p} R_{\text {out }}+C_{\text {out }} R_{\text {out }}\right)$ is the dominant pole of the LDR in the light load.

The unity-gain frequency is approximated as

$$
U G F_{1}=\frac{R_{F 2}}{R_{F 1}+R_{F 2}} \cdot \frac{g_{m 1}}{C_{a}} \cdot \frac{1}{1+\left(C_{\text {out }} / C_{a} R_{1} g_{m 2} R_{2} g_{m p}\right)}
$$

For the light load, two zeros, $1 / R_{\text {esr }} C_{\text {out }}$ and $g_{m 1} g_{m 2} / g_{m} C_{1}$, in (1) are much higher than the unity-gain frequency of (2) and their effects are neglected. From the second-order polynomial in the denominator of (1), the natural frequency and the damping factor are calculated as

$$
\begin{aligned}
\omega_{n} & =\sqrt{\frac{C_{a} g_{m 2} g_{m p}+\left(C_{\text {out }} / R_{1} R_{2}\right)}{C_{\text {out }} C_{1} C_{2}}} \text { and } \\
\zeta & =\frac{1}{2} \frac{C_{a} g_{m 2} g_{m p} R_{e s r}+\left(C_{2} / R_{1}\right)+\left(C_{1} / R_{2}\right)}{\left.\sqrt{C_{1} C_{2}\left(\left(C_{a} / C_{\text {out }}\right) g_{m 2} G_{m p}+\left(1 / R_{1} R_{2}\right)\right.}\right)}
\end{aligned}
$$

To guarantee the stability in the light load, the non-dominant poles should be higher than the unity-gain frequency, and the damping factor should be designed appropriately to ensure that the ESR and output capacitor do not affect the phase margin. From (2) and (3), the following constraint should be satisfied.

$$
\begin{aligned}
C_{\text {out }}> & \frac{1}{2} C_{a} g_{m 2} g_{m p} R_{1} R_{2} \\
& {\left[-1+\sqrt{1+4\left(\frac{g_{m 1}}{C_{a}}\right)^{2}\left(\frac{R_{F 2}}{R_{F 1}+R_{F 2}}\right)^{2} R_{1} R_{2} C_{1} C_{2}}\right] }
\end{aligned}
$$

Equation (4) indicates that there is a lower bound of the output capacitor in this proposed LDR. In this case, the output capacitor is designed to be around several $\mathrm{nF}$.

When the LDR is in the heavy load (i.e. $I_{\text {out }}$ is large), the loop transfer function is

$$
\begin{gathered}
-A_{d c}\left(1+s C_{z} R_{z}\right)\left(1+s C_{\text {out }} R_{e s r}\right) \\
L_{h}(s) \simeq \frac{\left(1+s\left(C_{a} / g_{m a}\right)\right)\left(1+s\left(g_{m f} C_{1} / g_{m 1} g_{m 2}\right)\right)}{\left(1+\left(s / P_{1} h\right)\right)\left(1+\left(s /\left(g_{m a} g_{m 2} g_{m p} R_{e s r}\left(R_{1} / / R_{z}\right) / C_{2}\right)\right.\right.} \\
\left.+\left(g_{m a} / C_{a}\right)\right)\left(1+s C_{1}+R_{z}\right)\left[1+s\left(C_{z} R_{z}+C_{\text {out }} R_{e s r}\right)\right. \\
+s^{2}\left(C_{z} R_{z} C_{\text {out }} R_{e s r}+\left(\left(C_{z} R_{z}\right.\right.\right. \\
\left.\left.\left.\left.+C_{z} R_{1}\right) C_{2} C_{\text {out }} / C_{a} R_{1} g_{m 2} g_{m p}\right)\right)\right]
\end{gathered}
$$

Five poles and four zeros are given in (5). The dominant pole is $p_{1 \_} h=1 / C_{a} R_{1} g_{m 2} R_{2} g_{m p} R_{\text {out }}$ and the unity-gain frequency in the heavy load is $U G F_{h}=R_{F 2} g_{m l} /\left(R_{F 1}+R_{F 2}\right) C_{a}$. For the heavy load, two zeros, $g_{m a} / C_{a}$ and $g_{m 1} g_{m 2} / g_{m j} C_{1}$, and two non-dominant poles, $\left(\left(g_{m a} g_{m 2}\right.\right.$ $\left.g_{m p} R_{\text {ear }}\left(R_{l} / / R_{z}\right) / C_{2}\right)+\left(g_{m a} / C_{a}\right)$ and $1 / R_{z} C_{1}$, in (5) are much higher than the unity-gain frequency of $U G F_{-} h$ and their effects are neglected. Assume $R_{e s r}$ is large and the following equation is satisfied:

$$
R_{e s r} \gg \frac{C_{2}}{C_{a}\left(R_{1} / / R_{z}\right) g_{m 2} g_{m p}}
$$

According to (6), the second-order polynomial in the denominator of (5) can be decomposed as $\left(1+s C_{z} R_{z}\right)\left(1+s C_{\text {out }} R_{\text {esr }}\right)$. Then, two pole-zero pairs can be eliminated. Therefore, the stability of this LDR in the heavy load is ensured when $R_{e s r}$ is large.

When $R_{e s r}$ is small, one of the poles in the second-order polynomial in the denominator of (5) is shifted to a lower frequency. This pole is expressed as

$$
P_{\text {nondom_h_lowESR }}=\frac{1}{C_{\text {out }} R_{e s r}+\left(C_{2} C_{\text {out }} / C_{a} g_{m 2} g_{m p}\left(R_{1} / / R_{z}\right)\right)}
$$

To ensure stability, this non-dominant pole must be higher than the unity-gain frequency. It results in the following constraint as 


$$
C_{\text {out }}<\frac{R_{F 1}+R_{F 2}}{R_{F 2}} \cdot \frac{C_{a}}{g_{m 1}} \cdot \frac{1}{R_{e s r}+\left(C_{2} / C_{a} g_{m 2} g_{m p}\left(R_{1} / / R_{z}\right)\right)}
$$

Equation (8) shows the upper bound of the output capacitor. Since this LDR only has a dominant pole within the unity-gain frequency, stability is guaranteed in the heavy load when $R_{e s r}$ is small. Based on (4) and (8), the allowable range of the output capacitor and the ESR for the LDR will be extended.

To verify the stability of the proposed LDR, Fig. 2 shows its root-locus plot with varying output currents. When the constraints in (4) and (8) are satisfied, the solid line shows that all the poles of the proposed LDR lie in the left-hand plane with the output current of $I_{\text {out }}=0 \mathrm{~mA}-I_{\text {out }}=150 \mathrm{~mA}$. When the constraints in (4) and (8) are not satisfied, the dotted line shows the complex poles move into the righthand plane with the output current of $I_{\text {out }}=0.3 \mathrm{~mA}-I_{\text {out }}=30 \mathrm{~mA}$. It results in an unstable system as the output current varied.

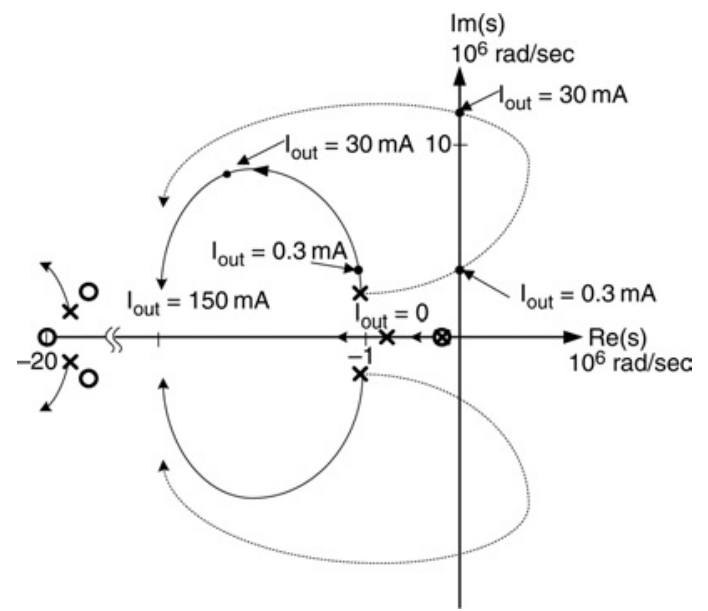

Fig. 2 Root-locus of proposed $L D R$

Arrows indicate the direction, which the output current increases Solid line: (4) and (8) satisfied; dotted line: (4) and (8) not satisfied

Experimental results: To verify the above analysis, the proposed LDR with a bandgap reference has been fabricated in a 0.35 um CMOS process.

For the proposed LDR, the measured line regulations at the output current $\mathrm{I}_{\text {out }}=0$ and $\mathrm{I}_{\text {out }}=150 \mathrm{~mA}$ are 0.134 and $0.100 \% / \mathrm{V}$, respectively. The measured maximum load regulation is $92.54 \mathrm{ppm} / \mathrm{mA}$ at $\mathrm{V}_{\text {in }}=3.3 \mathrm{~V}$. Fig. 3 shows the measured transient response of the LDR at the supply of $3.3 \mathrm{~V}$ under the output current switching from 0 to 20, 50, and $150 \mathrm{~mA}$. Fig. $3 a$ shows the result for $C_{\text {out }}=47 \mu \mathrm{F}$ with $R_{e s r}=50 \Omega$ and Fig. $3 b$ shows the result for $C_{\text {out }}=2 \mathrm{nF}$ with $R_{e s r}=0.1 \Omega$. It shows that this LDO can recover the output voltage within $20 \mu$ s within the error of $0.5 \%$ for switching the output current of $150 \mathrm{~mA}$.
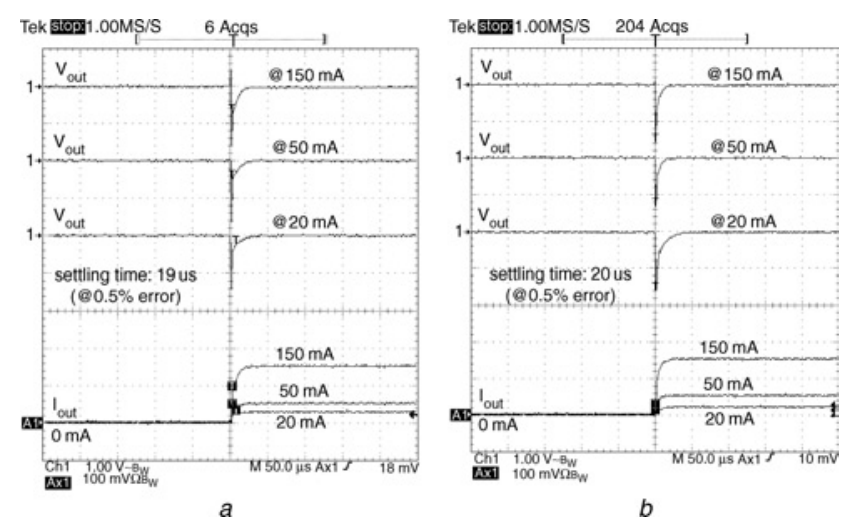

Fig. 3 Transient response of $L D R$ for $C_{\text {out }}=47 \mu F$ and $R_{\text {esr }}=50 \Omega$

(Fig. 3a) $C_{\text {out }}=2 \mathrm{nF}$ and $R_{\text {esr }}=0.1 \Omega$ (Fig. 3b) $V_{\text {out }}: 500 \mathrm{mV} /$ div., $10 u s /$ div.

Conclusions: A CMOS LDR with a dynamic zero compensation is presented. The experimental results confirm that this LDR with dynamic zero compensation can be stable for a wide range of the output capacitor and the ESR.

(C) The Institution of Engineering and Technology 2007

13 April 2007

Electronics Letters online no: 20071063

doi: 10.1049/el:20071063

C.-L. Chen, W.-J. Huang and S.-I. Liu (Graduate Institute of Electronics Engineering and Department of Electrical Engineering, National Taiwan University, Taipei, Taiwan 10617, Republic of China)

E-mail: 1si@cc.ee.ntu.edu.tw

\section{References}

1 Leung, K.N., and Mok, P.K.T.: 'A capacitor-free CMOS low-dropout regulator with damping-factor-control frequency compensation', IEEE J. Solid-State Circuits, 2003, 38, pp. 1691-1702

2 Hazucha, P., Karnik, Y., Bloechel, B., Parsons, C., Finan, D., and Borkar, S.: 'An area-efficient integrated linear regulator with ultra-fast load regulation'. Dig. Tech. Pprs, Symposium on VLSI Circuits, June 2004 pp. 218-221

3 Huang, W.J., Lu, S.H., and Liu, S.I.: 'A CMOS low dropout linear regulator with single Miller capacitor', Electron. Lett., 2006, 42, pp. 216-217

4 Bang Lee, S.: 'Technical review of low dropout voltage regulator operation and performance', (Application Reports, Texas Instruments Inc., Literature number SLVA072)

5 Lee, H., and Mok, P.K.T.: 'Advances in active-feedback frequency compensation with power optimization and transient improvement', IEEE Trans. Circuits Syst. I, 2004, 51, pp. 1690-1696

6 Ahuja, B.K.: 'An improved frequency compensation technique for CMOS operational amplifiers', IEEE J. Solid-State Circuits, 1983, 18, pp. 629-633 